\title{
Los estilos educativos paternos y maternos en la adolescencia y su relación con la resiliencia, el apego y la implicación en acoso escolar
}

\author{
Olga Gómez-Ortiz ${ }^{1 *}$, Rosario Del Rey ${ }^{2}$, Eva-María Romera ${ }^{1}$ y Rosario Ortega-Ruiz ${ }^{1,3}$ \\ 1 Universidad de Córdoba (España). \\ 2 Universidad de Sevilla (España). \\ 3 University of Greenwich (Reino Unido).
}

\begin{abstract}
Resumen: El primer objetivo de este trabajo es establecer una clasificación de estilos parentales y el segundo examinar la relación entre los estilos materno, paterno y la coherencia entre ambos, y el ajuste adolescente, evaluado mediante la resiliencia, el apego y la implicación en bullying. Para ello, una muestra incidental de 626 estudiantes $(49.7 \%$ chicas) de educación secundaria de la provincia de Córdoba, cumplimentaron la Escala para la evaluación del estilo educativo de padres y madres de adolescentes, el European Bullying In tervention Project Questionaire, la escala de apego CaMir-R y la versión reducida de la Escala de Resiliencia de Connor y Davidson. Los resultados muestran cuatro estilos educativos análogos para madres y padres ("democrático controlador", "democrático supervisor", "democrático de baja revelación" y "moderado"), un estilo "indiferente" solo hallado en la clasificación paterna y un estilo "permisivo" solo observado en la categorización materna Asimismo, se observan diferencias significativas en ajuste adolescente en función del estilo paterno y materno y la coherencia entre ambos, reflejando el mejor ajuste los hijos de padres o madres "democráticos supervisores" y los que ambos progenitores eran democráticos.

Palabras clave: Familia; estilos educativos; adolescencia; coherencia parental; ajuste psicológico; bullying; resiliencia; apego.
\end{abstract}

Title: Maternal and paternal parenting styles in adolescence and its relationship with resilience, attachment and bullying involvement.

Abstract: The present research has two aims. The first is to create a typology of parenting style, and the second is to explore the relationship between mother's and father's parenting styles and the coherence between both, and adolescent adjustment, assessed with the bullying involvement, resilience and attachment. It has been used an incidental sample of 626 high school students $(49.7 \%$ girls) from Córdoba, who completed the Scale to assessment maternal and paternal parenting style in adolescence, the European Bullying Intervention Project Questionnaire, the attachment scale CaMir-R and the short version of the Connor-Davidson Resilience Scale. The results show four parenting styles, which are the same for mothers and fathers ("supervisor democratic", "controlling democratic", "democratic of little disclosure" and "moderately") and one parenting style only for mothers ("permissive") and other, only for fathers ("indifferent"). It was found statistically significant differences in all measures of adolescent adjustment depending on mother's and father's parental styles and the coherence between both. The better psychosocial adjustment was observed in adolescents whose father or mother were supervisor democratic and when both parents were democratic.

Key words: Family; parenting styles; adolescence; parental coherence; psychological adjustment; bullying; resilience; attachment.

vo, rechazante, suficiente y autoritativo), que ofrecían una elevada singularidad y riqueza informativa.

Actualmente, las investigaciones sobre estilos educativos parentales están abandonando en parte este enfoque tipológico, para centrarse en una perspectiva dimensional, desde la que se analizan un mayor número de dimensiones parentales, que permiten captar no solo la mayor variabilidad de los estilos educativos parentales, sino también la bidireccionalidad existente entre las conductas de los progenitores y las de los propios hijos e hijas (Oliva, Parra, Sanchez-Queija, \& López, 2007). A este respecto, los estudios que han analizado la relación entre las dimensiones de los estilos parentales, encuentran una elevada relación entre el afecto y la promoción de autonomía, la auto-revelación y el humor; mientras que el afecto y el control conductual y este último y otras formas de control, como el psicológico y la auto-revelación, aparecen escasamente relacionados (García \& Gracia, 2009, 2010; Oliva et al., 2007). Otros autores (Silk, Morris, Kanaya, \& Steinberg, 2003) resaltan una importante relación entre la autonomía psicológica y el control conductual, calificando al control psicológico como un constructo independiente, de escasa relación con las otras dos dimensiones.

Desde esta perspectiva y en base a los resultados de un análisis de conglomerados (clúster) con las dimensiones de afecto y comunicación, control conductual, promoción de autonomía, humor, control psicológico y revelación de los hijos hacia ambos progenitores, Oliva, Parra y Arranz (2008) encontraron tres estilos educativos parentales fundamentales, 
tanto en las madres como en los padres (democrático, estricto e indiferente). Dicha categorización, aunque guardaba una estrecha relación con las tipologías tradicionales, permitió conocer con mayor profundidad qué otras dimensiones, además del afecto y el control, caracterizaban los estilos educativos parentales establecidos en la misma y la relación que guardaban estos con el ajuste adolescente. En este sentido, se demostró que el estilo democrático, además de ser el más frecuente, era el que más beneficiaba a los jóvenes, en términos de menores problemas internalizantes y externalizantes y mayor desarrollo positivo, satisfacción vital y autoestima. Estos resultados son congruentes con los de otras investigaciones, en los que se ha demostrado la mayor prevalencia del estilo democrático, comparada con la de los otros estilos (Hoeve, Dubas, Gerris, Van del Laan, \& Smeenk, 2011; Oliva, et al., 2008; Torío, Peña, \& Rodríguez, 2008; Wolfradt, Hempel, \& Miles, 2003) y su beneficio en el ajuste adolescente a través de la prevención de delincuencia (Deutsch, Crockett, Wolff, \& Russell, 2012; Hoeve, et al., 2011) y de la implicación en acoso escolar (Baldry \& Farrington, 2005; Gómez-Ortiz, Del Rey, Casas, \& OrtegaRuiz, 2014; Kawabata, Alink, Tsen, Van Ijzendoorn, \& Crick, 2011), y el desarrollo de la autoestima (Alonso \& Román, 2005), el rendimiento académico (Besharata, Azizia, \& Poursharifib, 2011; Frey, Ruchkin, Martin, \& SchwabStone, 2009), la competencia social y capacidad resiliente (Baumrind, 1991; Masten \& Powell, 2003). Asimismo, los estudios realizados parecen sugerir la relación entre unas prácticas parentales coherentes con el estilo democrático y una mayor probabilidad de desarrollar un apego seguro (Cantón \& Cortés, 2005), subyaciendo a esta última relación todos los beneficios adaptativos que se atribuyen a la generación de este tipo de apego.

Sin embargo, otros estudios ponen de manifiesto la superioridad del estilo permisivo o indulgente en relación a la promoción del buen ajuste infanto-juvenil, especialmente en culturas colectivistas-horizontales como la española (Fuentes, García, Gracia, \& Alarcón, 2015; García \& Gracia, 2009, 2010; Pérez, 2012)

En cuanto al ajuste psicológico del adolescente, otra línea de investigaciones sobre los estilos educativos parentales, es aquella que analiza la percepción de diferencias en los estilos educativos del padre y la madre. En este sentido, se ha demostrado que la situación más favorable para los hijos e hijas, la que se relaciona con menores problemas psicopatológicos y mayor desarrollo positivo, se da cuando ambos padres son democráticos, o en su defecto, cuando alguno de los progenitores presenta dicho estilo, siendo la peor combinación, el que ambos padres fueran negligentes, situación que favorecía especialmente la implicación en delincuencia (Berkien, Louwerse, Verhulst, \& Van der Ende, 2012; Hoeve, et al., 2011; Oliva et al., 2008; Steinberg, 2001). No se ha encontrado, sin embargo, evidencia empírica anterior, que analice la relación entre la coherencia parental y otros indicadores de ajuste ajenos a la psicopatología o la delincuencia.
Con todo lo dicho hasta aquí, parece claro que aunque ha habido un notable desarrollo teórico sobre la naturaleza de las conductas y actitudes educativas parentales, es necesario continuar investigando los estilos educativos en que estas se enmarcan y su relación con el ajuste adolescente, pues en los últimos años, posiblemente se han producido importantes cambios en la vida de las familias que podrían haber modificado algunos de los supuestos teóricos de las investigaciones clásicas.

Este estudio se centrará, por tanto, en la exploración de las agrupaciones en que se organizan los estilos educativos parentales, y muy particularmente el estilo democrático, ya que es importante conocer la variabilidad de conductas y actitudes educativas que se enmarcan dentro de este estilo tan prevalente, así como las dimensiones parentales que más favorecen la adaptación psicosocial en la adolescencia.

Para lograr este propósito, se entiende que lo más adecuado, quizás sea alejarse del enfoque tipológico o clasificatorio, y partir de un enfoque dimensional, centrado no tanto en la clasificación como en la descripción del estilo parental, a través de numerosas variables que ofrezcan una información más rica sobre los comportamientos y actitudes parentales evaluados y permitan recoger la variabilidad de los estilos educativos parentales actuales, y muy particularmente, el impacto que en el ajuste adolescente generan dichos estilos y la mayor o menor homogeneidad de los estatus parentales entre la madre y el padre. Un impacto que no será evaluado atendiendo a la psicopatología, sino en base a otros aspectos de la adaptación psicosocial escasamente investigados en relación con los estilos educativos parentales, como son la implicación en acoso escolar, el apego y la resiliencia.

En síntesis, los objetivos de esta investigación, han sido los siguientes:

1) Explorar las dimensiones parentales evaluadas y su agrupación, con el fin de establecer, si es necesario, una nueva tipología de estilos educativos adaptada a la realidad actual.

2) Analizar la relación entre los estilos educativos materno y paterno y la adaptación o ajuste psicosocial de los adolescentes, evaluado en función de la implicación en acoso escolar, del apego que desarrollan hacia sus padres y madres y de su capacidad resiliente.

3) Examinar la relación entre la coherencia entre el estilo educativo materno y paterno y el ajuste psicosocial de los adolescentes.

Basándonos en los estudios citados anteriormente, planteamos la hipótesis, de que existirá una relación significativa entre los estilos parentales y su coherencia en la madre y el padre, y el ajuste adolescente. Concretamente pensamos que los estilos democráticos serán los que más favorezcan el ajuste adolescente, sobre todo los que sean bajos en control psicológico; y que los mejores resultados en adaptación psicosocial aparecerán en los jóvenes cuyos progenitores sean ambos democráticos. 


\section{Método}

\section{Participantes}

La muestra incidental estuvo compuesta por 626 estudiantes $(49.7 \%$ chicas) de todos los cursos de Educación Secundaria Obligatoria (ESO) (el 25\% cursaban $1^{\circ}$ de la ESO; el $28.3,2^{\circ}$, el $22.6 \%, 3^{\circ}$ y el $\left.24.2^{\%}, 4^{\circ}\right)$. Dichos estudiantes pertenecían a cuatro institutos de Educación Secundaria de Córdoba, y sus edades estaban comprendidas entre los 12 y los 18 años $(M=14.1 ; D . T .=1.38)$, habiendo nacido la mayoría de los mismos en España (97.6\%).

\section{Instrumentos}

La implicación en bullying se evaluó a través del European Bullying Intervention Project Questionnaire (EBIPQ; Brighi et al., 2012). Autoinforme compuesto por 14 ítems tipo Likert de cinco opciones de respuesta ("no", "sí, una o dos veces", "sí, una o dos veces al mes", "sí, alrededor de una vez a la semana" y "sí, más de una vez a la semana"). Siete de estos ítems estaban referidos a conductas de agresión y otros siete a situaciones victimización entre iguales, de naturaleza verbal, física y relacional, directa e indirecta. El tiempo de referencia para evaluar la frecuencia de implicación ha sido los últimos seis meses. Los índices de consistencia interna, Alfa de Cronbach, fueron adecuados ( $\alpha$ victimización $=.78$; aagresión $=.75 \mathrm{y} \alpha$ total $=.82$ ).

Los estilos educativos parentales se han evaluado utilizando la Escala para la evaluación del estilo educativo de padres y madres de adolescentes (Oliva, et al., 2007), compuesta por 82 ítems tipo Likert de seis opciones de grado de acuerdo (41 para el estilo educativo del padre, y 41 para el de la madre). Esta escala valora seis dimensiones: A) afecto y comunicación: percepción de apoyo y de relaciones cercanas entre padres e hijos e intercambio de puntos de vista de forma clara, respetuosa, afectiva y empática; B) promoción de la autonomía: grado en que los padres fomentan la independencia y la capacidad de tomar decisiones de su hijo o hija; C) control conductual: conducta paterna de poner límites y supervisar la conducta filial; D) control psicológico: supone el uso de estrategias intrusivas y manipuladoras cuando el adolescente hace algo que los padres no aprueban; E) auto-revelación: tendencia del adolescente a informar espontáneamente a sus padres sobre lo que hace cuando no está con ellos; F) humor: actitud parental relajada, alegre y optimista. En este estudio, la consistencia interna, evaluada a través del Alfa de Cronbach, ha sido buena, tanto a nivel general ( $\alpha$ total = $.941)$, como en las distintas escalas $(\alpha \mathrm{A}$ madre $=.89 ; \alpha \mathrm{A}$ padre $=.90 ; \alpha \mathrm{B}$ madre $=.82 ; \alpha \mathrm{B}$ padre $=.84 ; \alpha \mathrm{C}$ madre $=.78$; $\alpha \mathrm{C}$ padre $=.81 ; \alpha \mathrm{D}$ madre $=.83 ; \alpha \mathrm{D}$ padre $=.81 ; \alpha \mathrm{E}$ madre $=.81 ; \alpha \mathrm{E}$ padre $=.84 ; \alpha \mathrm{F}$ madre $=.87 ; \alpha \mathrm{F}$ padre $=.86)$.

La Escala de Resiliencia de Connor y Davidson (2003), en su versión reducida, ha sido el instrumento de elección para evaluar la resiliencia. Este instrumento ha sido validado en población española por Notario-Pacheco et al., (2011) y consta de 10 ítems tipo likert, que ofrecen 5 alternativas de respuesta $(0=$ en absoluto y $4=$ casi siempre). Esta escala está compuesta por un único factor, que mostró una adecuada consistencia interna $(\alpha=.75)$.

Para evaluar el apego se utilizaron algunas escalas de una versión reducida de la escala CaMir-R (Balluerka, Lacasa, Gorostiaga, Muela, \& Pierrehumbert, 2011). El formato de respuesta es de tipo likert y se ofrecen cinco alternativas para cuantificar el grado de acuerdo. Las escalas que se utilizaron, fueron las denominadas: Seguridad, disponibilidad y apoyo de las figuras de apego (-SDA- representa al apego seguro y hace referencia a la percepción de sentir amor, confianza y disponibilidad en las figuras de apego), Preocupación familiar (-PF- alude a la preocupación actual por los seres queridos y al sentimiento de ansiedad por separación de los mismos y representa al apego preocupado) y Traumatismo infantil (-TI- representa al apego desorganizado y hace referencia a los recuerdos infantiles de falta de disponibilidad, violencia y amenazas de las figuras de apego). Dichas escalas mostraron una adecuada fiabilidad $\left(\alpha_{\mathrm{SDA}}=.83 ; \alpha_{\mathrm{PF}}=.67 ; \alpha_{\mathrm{TI}}=.67\right)$.

\section{Procedimiento}

La investigación tiene un diseño transversal, ex post facto retrospectivo, un grupo, múltiples medidas (Montero \& León, 2007). Tras obtener los permisos oportunos por parte de los equipos directivos de los institutos de educación secundaria, se visitaron los centros para administrar el cuestionario. Antes de ello, se insistió al alumnado sobre el carácter anónimo, confidencial y voluntario de su participación. El tiempo medio de cumplimentación de la batería osciló entre 45 minutos y una hora.

\section{Análisis de datos}

En primer lugar se realizó un análisis de correlaciones entre las dimensiones de los estilos educativos para conocer el grado de relación existente entre las mismas previamente al establecimiento de grupos basado en las relaciones entre dichas dimensiones. A continuación se llevó a cabo un análisis de conglomerados jerárquico con todas las variables referidas al estilo educativo de la madre y otro con las relativas al estilo del padre, con el fin de determinar el número correcto de conglomerados o clúster que representa la estructura interna de los estilos educativos. Se usó el método Ward para minimizar las diferencias dentro de cada conglomerado y evitar el problema de las largas cadenas de observaciones

La clasificación de los estilos educativos parentales, se elaboró a partir de un análisis de conglomerados por el procedimiento $K$ medias. En este caso, no realizamos un análisis de conglomerados jerárquico para evitar la creación de clúster compuestos por un número similar de individuos, y la imposición de una estructura jerárquica al constructo, sin existir evidencia empírica al respecto.

Para confirmar la solución ofrecida por el análisis de conglomerados, llevamos a cabo un análisis discriminante 
predictivo, utilizando como variables predictoras el conjunto de variables que representan las dimensiones de los estilos educativos parentales y como variable de clasificación, el conglomerado de pertenencia. Se utilizó el método de validación cruzada, con el fin de reducir el sesgo que se produce cuando los mismos sujetos son utilizados para computar los coeficientes con los que posteriormente serán clasificados (Cea d'Ancona, 2004).

Asimismo, se han realizado análisis de la varianza (ANOVA) para evaluar la relación entre el estilo educativo paterno y materno y la adaptación psicosocial del adolescente y para examinar la relación entre la coherencia entre el estilo educativo materno y paterno y cada una de las variables que representan la adaptación psicosocial. Para analizar las diferencias de medias entre los grupos, se utilizó la prueba post hoc de Tukey y Games-Howell. Las diferencias significativas obtenidas con el ANOVA fueron contrastadas con los resultados derivados de la medida del tamaño del efecto (D de Cohen). Un valor en este índice menor o igual a .20 indicaría un efecto pequeño, mientras que valores superiores a .20 y hasta .50 mostrarían un efecto moderado y superiores a .80 indicarían un efecto elevado (Cohen, 1992). El nivel de significatividad adoptado para realizar los contrastes de medias fue de $p<.01$. La codificación y el análisis de datos se realizaron con el paquete estadístico SPSS versión 18.0.

\section{Resultados}

\section{La clasificación de los estilos educativos materno y paterno}

Las correlaciones de Pearson entre las dimensiones de los estilos educativos de la madre y del padre indicaron que la mayor parte de estas dimensiones se encontraban relacionadas de forma significativa con las demás. Destaca, en sentido positivo, la relación entre afecto y comunicación, promoción de autonomía, revelación y humor y la correlación entre control conductual y revelación, todo ello en ambos progenitores. También correlacionan significativamente, aunque con valores poco elevados, el control conductual con el control psicológico y el humor y el afecto y comunicación con el control conductual, especialmente en el caso de la madre. Por otro lado, destaca la relación inversa entre control psicológico, por un lado, y afecto y comunicación, promoción de autonomía y humor, por otro, tanto en el padre como en la madre. No se observa relación significativa entre control psicológico y revelación en ambos padres, y entre promoción de autonomía y control psicológico, en el caso del padre (ver Tabla 1).

Tabla 1. Correlaciones de Pearson entre las variables del estilo educativo materno y paterno.

\begin{tabular}{|c|c|c|c|c|c|c|c|c|c|c|c|c|}
\hline & $\begin{array}{c}\text { 1. AC } \\
\mathrm{M}\end{array}$ & $\begin{array}{c}\text { 2. PA } \\
\mathrm{M}\end{array}$ & $\begin{array}{c}\text { 3. CC } \\
\mathrm{M}\end{array}$ & $\begin{array}{c}\text { 4. CP } \\
\mathrm{M}\end{array}$ & $\begin{array}{c}\text { 5. REV } \\
\text { M }\end{array}$ & $\begin{array}{c}\text { 6. HUM } \\
\text { M }\end{array}$ & $\begin{array}{l}\text { 7. } \mathrm{AC} \\
\mathrm{P}\end{array}$ & $\begin{array}{l}\text { 8. PA } \\
\text { P }\end{array}$ & $\begin{array}{c}\text { 9. } \mathrm{CC} \\
\mathrm{P}\end{array}$ & $\begin{array}{c}\text { 10. CP } \\
\mathrm{P}\end{array}$ & $\begin{array}{l}\text { 11. REV } \\
\text { P }\end{array}$ & $\begin{array}{l}\text { 12. HUM } \\
\text { P }\end{array}$ \\
\hline 1 & 1 & & & & & & & & & & & \\
\hline 2 & $.612^{* * *}$ & 1 & & & & & & & & & & \\
\hline 3 & $.210^{* * *}$ & $.286^{* * *}$ & 1 & & & & & & & & & \\
\hline 4 & $-.224^{* * *}$ & $-.152^{* * *}$ & $.284^{* * *}$ & 1 & & & & & & & & \\
\hline 5 & $.492^{* * *}$ & $.476^{* * *}$ & $.357^{* * *}$ & -.079 & 1 & & & & & & & \\
\hline 6 & $.664^{* * *}$ & $.588^{* * *}$ & $.147^{* * *}$ & $-.217^{* * *}$ & $.444^{* * *}$ & 1 & & & & & & \\
\hline 7 & $.608^{* * *}$ & $.417^{* * * *}$ & $.168^{* * *}$ & $-.139^{* *}$ & $.321^{* * *}$ & $.464^{* * *}$ & 1 & & & & & \\
\hline 8 & $.458^{* * *}$ & $.796^{* * * *}$ & $.256^{* * * *}$ & -.081 & $.375^{\text {**** }}$ & $.439^{* * * *}$ & $.612^{* * *}$ & 1 & & & & \\
\hline 9 & $.228^{* * *}$ & $.270^{* * * *}$ & $.782^{* * *}$ & $.228^{* * * *}$ & $.378^{* * *}$ & $.189^{* * * *}$ & $.364^{* * *}$ & $.367^{* * * *}$ & 1 & & & \\
\hline 10 & $-.125^{* *}$ & -.071 & $.263^{* * *}$ & $.851^{* * *}$ & .011 & $-.121^{* *}$ & $-.137^{* *}$ & -.081 & $.293^{* * * *}$ & 1 & & \\
\hline 11 & $.363^{* * *}$ & $.343^{* * *}$ & $.358^{* * *}$ & .014 & $.777^{* * * *}$ & $.465^{* * *}$ & $.549^{* * *}$ & $.491^{* * *}$ & $.496^{* * *}$ & .055 & 1 & \\
\hline 12 & $.425^{* * * *}$ & $.382^{* * *}$ & $.176^{* * *}$ & -.068 & $.444^{* * *}$ & $.607^{* * * *}$ & $.653^{* * *}$ & $.567^{* * *}$ & $.176^{* * *}$ & $-.119^{* *}$ & $.348^{* * * *}$ & 1 \\
\hline
\end{tabular}

Nota AC = Afecto y comunicación; PA = Promoción de autonomía; CC = Control conductual; CP = Control psicológico; REV. $=$ Auto-revelación; HUM. = Humor; $\mathrm{M}=$ Madre; $\mathrm{P}=$ Padre; ${ }^{*}=p<.01 ; * * *=p<.001$

Los resultados del dendrograma y del historial de conglomeración, obtenidos a partir del análisis de conglomerados jerárquico, revelaron que las soluciones de 2, 3, 4 y 5 clúster serían adecuadas, tanto para la madre, como para el padre. Para decidir el número óptimo de clúster, se examinó la distancia entre conglomerados a cada paso sucesivo, definiendo el clúster los grandes saltos entre los valores sucesivos del coeficiente de aglomeración, que implicaban, que añadir el siguiente caso al conglomerado, supondría una pérdida de homogeneidad en el mismo

En el análisis de conglomerados por el procedimiento $K$ medias, se solicitó la determinación de 5 conglomerados para ofrecer una clasificación de los estilos educativos que lograra captar la mayor variabilidad posible de este constructo, ma- ximizado la homogeneidad intra-grupo y la heterogeneidad inter-grupos. Todos los grupos establecidos a partir de este análisis, excepto uno de ellos, fueron análogos en el padre y en la madre.

Los padres y madres del primer grupo obtuvieron una elevada puntuación en las dimensiones afecto y comunicación, humor, auto-revelación y promoción de la autonomía y las máximas puntuaciones de todos los grupos en las dimensiones control conductual y psicológico. Estos padres y madres fueron denominados "democráticos controladores". Los padres y madres del segundo grupo, que mostraron las puntuaciones más elevadas de todos los grupos en afecto y comunicación, promoción de autonomía, auto-revelación materna y humor, unas elevadas puntuaciones en control 
conductual -aunque menores que las del primer grupo- y las puntuaciones más bajas de todos los grupos en control psicológico, fueron denominados "democráticos supervisores". El tercer grupo de padres y madres, presentó unas puntuaciones moderadas en control psicológico y elevadas en las dimensiones de afecto y comunicación, promoción de autonomía, humor, y control conductual, aunque menores que las obtenidas por los componentes de los grupos anteriores, siendo mucho menores las puntuaciones en auto-revelación. Este grupo fue denominado "democrático de baja revelación”. El cuarto grupo de padres y madres se caracterizó por presentar las puntuaciones más bajas de los grupos hasta aquí mencionados, en todas las dimensiones, excepto en el control psicológico. Puesto que dichas puntuaciones, no eran demasiado elevadas, ni demasiado bajas (oscilaban entre 2.15 y 3.77 en una escala likert 1-6), se denominó a este grupo "moderados". El último grupo solo de padres, obtuvo bajas puntuaciones en todas las dimensiones. Puntuaciones que fueron aún más bajas que las del grupo de padres y madres "moderados", excepto las de la dimensión control psicológico, que fueron similares. A estos padres les fue administrada la etiqueta de "indiferentes". El quinto grupo referido al estilo educativo materno, se caracterizó por presentar puntuaciones elevadas en afecto, promoción de la autonomía y humor y puntuaciones moderadas hacia bajas en las dimensiones relativas al control y a la auto-revelación. La etiqueta de madres "permisivas" fue considerada apropiada para este grupo. En la Tabla 2 pueden observarse los centros de los conglomerados finales en cada dimensión para todos los grupos y el número de casos categorizados en cada grupo.

Tabla 2. Centros de los conglomerados finales y tamaño de cada grupo establecido en el análisis de conglomerados.

\begin{tabular}{|c|c|c|c|c|c|c|c|c|c|c|}
\hline & DC M & DC P & DS M & DS P & DBR M & DBR P & MOD M & MOD P & PER & I \\
\hline$\overline{\mathrm{AC}}$ & 5.47 & 5.38 & 5.70 & 5.56 & 4.91 & 4.90 & 3.46 & 3.70 & 5.28 & 2.21 \\
\hline PA & 5.06 & 5.07 & 5.26 & 5.21 & 4.46 & 4.64 & 2.81 & 3.73 & 4.55 & 1.90 \\
\hline $\mathrm{CC}$ & 5.27 & 5.25 & 4.87 & 4.45 & 4.78 & 3.93 & 3.73 & 3.22 & 2.81 & 2.49 \\
\hline $\mathrm{CP}$ & 4.39 & 4.06 & 2.14 & 1.78 & 3.33 & 2.86 & 3.66 & 3.02 & 2.28 & 3.05 \\
\hline REV. & 4.95 & 4.86 & 5.16 & 4.75 & 3.09 & 2.88 & 2.52 & 2.15 & 3.17 & 1.51 \\
\hline HUM. & 5.05 & 5.29 & 5.39 & 5.54 & 4.34 & 5.03 & 2.61 & 3.77 & 4.95 & 2.64 \\
\hline $\mathrm{N}$ & 109 & 105 & 164 & 98 & 124 & 160 & 45 & 96 & 82 & 17 \\
\hline
\end{tabular}

Nota. DC $=$ Democrático controlador; DS = Democrático supervisor; DBR = Democrático de baja revelación; MOD = Moderado; PER = Permisivo; I = Indiferente; $\mathrm{N}=\mathrm{n}^{\circ}$ de casos categorizados en cada grupo

Los resultados derivados del análisis de conglomerados apuntaron que, en el caso del estilo educativo de la madre, el $96.6 \%$ de los casos había sido correctamente categorizado. Concretamente, para el conglomerado permisivo, la tasa de identificación correcta fue $96.3 \%$; para el "democrático de baja revelación" fue $94.4 \%$; para el democrático controlador fue $96.3 \%$; para el moderado fue $100 \%$ y para el conglomerado democrático supervisor, la tasa de clasificación correcta fue $97.6 \%$.

Para valorar el éxito de la clasificación en los estilos educativos maternos, se contrastó el porcentaje global de casos correctamente clasificados, con el porcentaje esperado (porcentaje de casos que podría clasificarse correctamente por casualidad, sin la ayuda de la función discriminante), denominado criterio de causalidad máxima cuando los grupos tienen un tamaño diferente. Dicho criterio se calcula dividiendo el número de casos que conforman el grupo de mayor tamaño por la muestra total, habiendo de ser el porcentaje global de aciertos, superior, al menos en un $25 \%$, al que se alcanzaría por mera casualidad (Cea d'Ancona, 2004). En nuestra muestra el criterio de casualidad máxima es $31.3 \%$ (164/524). Puesto que el porcentaje global de casos correctamente clasificados $(96.6 \%)$ es superior al $25 \%$ del criterio de causalidad máxima $(31.3+25 \%=39.1 \%)$, podemos confirmar el éxito del proceso de clasificación.

En cuanto al estilo educativo del padre, los resultados del análisis discriminante muestran que el $94.1 \%$ de los casos habían sido correctamente categorizados. Concretamente, para el conglomerado indiferente, la tasa de identificación correcta fue $94.1 \%$; para el democrático de baja revelación, fue $95 \%$; para el democrático controlador, fue $88.6 \%$; para el moderado, fue $96.9 \%$ y para el último conglomerado, "democrático supervisor", la tasa de clasificación correcta fue 95.9\% . En este caso, el criterio de casualidad máxima fue $33.6 \%$ (160/476). Puesto que el porcentaje global de aciertos $(93.6 \%)$ es claramente superior, al $25 \%$ del porcentaje que se alcanzaría por mera casualidad $(42 \%)$, se confirma el éxito del proceso de clasificación.

Estilos educativos materno y paterno y ajuste psicosocial adolescente

$\mathrm{Al}$ analizar la relación entre el estilo educativo materno y la agresión, victimización, resiliencia y apego, los resultados de la prueba ANOVA (ver Tabla 3) mostraron la existencia de diferencias estadísticamente significativas entre los grupos, en la agresión y victimización, en la resiliencia, y en las dimensiones que representan al apego seguro, preocupado y desorganizado.

Las pruebas post hoc reflejaron que los jóvenes con madres democráticas supervisoras obtuvieron las menores puntuaciones en la dimensión agresión y victimización, comparados con los adolescentes cuyas madres fueron categorizadas dentro de los otros estilos democráticos y dentro del estilo moderado. Asimismo, el grupo de adolescentes con madres permisivas mostró las menores puntuaciones en victimización, comparado con el grupo de jóvenes con madres democráticas controladoras, democráticas de baja revelación y moderadas. 
En la dimensión que representa al apego seguro (seguridad $y$ disponibilidad de las figuras de apego), fueron los grupos de adolescentes cuyas madres presentaron el estilo democrático baja revelación y moderado, los que mostraron la menor puntuación, comparados con los que sus madres se enmarcaron dentro del estilo democrático controlador y supervisor. El grupo de jóvenes con madres moderadas también mostró una significativa menor puntuación en apego seguro, comparado con el de madres permisivas y democráticas de baja revelación, así como el grupo de adolescentes con madres permisivas, comparado con el de madres democráticas supervisoras.

Los jóvenes con madres democráticas controladoras y supervisoras, mostraron las mayores puntuaciones en la dimensión que representa al apego preocupado (preocupación familiar), comparados con los de madres moderadas, democráticas de baja revelación y permisivas.

En cuanto al traumatismo infantil (dimensión que representa el apego desorganizado), el grupo de jóvenes cuyas madres fueron categorizadas como permisivas, comparado con el de madres democráticas controladoras y moderadas, y el grupo de chicos y chicas con madres democráticas supervisoras, comparado con los dos grupos anteriores y con los hijos e hijas de madres democráticas de baja revelación, fueron los que obtuvieron la menor puntuación.

Finalmente, los adolescentes con madres democráticas supervisoras mostraron una significativa mayor capacidad resiliente, comparados con los de madres moderadas y democráticas de baja revelación.

Los resultados de la prueba ANOVA (ver Tabla 4) indicaron diferencias estadísticamente significativas entre los grupos de estilos educativos paternos, en la agresión y victimización, en la resiliencia, y en las dimensiones que representan al apego seguro, preocupado y desorganizado. Las pruebas post hoc, mostraron que los jóvenes cuyos padres ejercían el estilo democrático supervisor, presentaron la menor puntuación en agresión, comparados con aquellos cuyos padres fueron categorizados como democráticos de baja revelación y moderados. El grupo de jóvenes con padres democráticos supervisores también reflejó unos índices significativamente menores de victimización que los jóvenes con padres democráticos de baja revelación.

Tabla 3. Diferencias en la implicación en acoso escolar, en el tipo de apego y en la capacidad resiliente en función del estilo educativo materno.

\begin{tabular}{|c|c|c|c|c|c|c|c|c|c|}
\hline & Grupos & $N$ & G.L & Media & D. $T$ & $F$ & Sig. & Post Hoc & $D$ de Cohen \\
\hline \multirow[t]{5}{*}{ Agresión } & DC & 110 & 4,523 & .392 & .468 & 6.528 & $.000^{* * *}$ & $\mathrm{DS} \neq \mathrm{DBR}$ & -.60 \\
\hline & DS & 164 & 4,523 & .242 & .354 & 6.528 & $.000^{* * *}$ & $\mathrm{DS} \neq \mathrm{DC}$ & -.37 \\
\hline & DBR & 121 & 4,523 & .489 & .478 & 6.528 & $.000^{* * *}$ & $\mathrm{DS} \neq \mathrm{MOD}$ & -.57 \\
\hline & MOD & 47 & 4,523 & .455 & .448 & 6.528 & $.000^{* * *}$ & & \\
\hline & PER & 82 & 4,523 & .429 & .372 & 6.528 & $.000^{* * *}$ & & \\
\hline \multirow[t]{6}{*}{ Victimización } & $\mathrm{DC}$ & 110 & 4,523 & .710 & .705 & 7.069 & $.000^{* * *}$ & $\mathrm{DS} \neq \mathrm{DBR}$ & .39 \\
\hline & DS & 164 & 4,523 & ,447 & .464 & 7.069 & $.000^{* * *}$ & $\mathrm{DS} \neq \mathrm{DC}$ & -.46 \\
\hline & DBR & 121 & 4,523 & .645 & .571 & 7.069 & $.000^{* * *}$ & $\mathrm{DS} \neq \mathrm{MOD}$ & -.63 \\
\hline & MOD & 47 & 4,523 & .778 & .713 & 7.069 & $.000^{* * *}$ & $\mathrm{PER} \neq \mathrm{DC}$ & -.48 \\
\hline & PER & 82 & 4,523 & .429 & .400 & 7.069 & $.000^{* * *}$ & $\mathrm{PER} \neq \mathrm{DBR}$ & -.42 \\
\hline & & & & & & & & $\mathrm{PER} \neq \mathrm{MOD}$ & -.66 \\
\hline \multirow{7}{*}{$\begin{array}{l}\text { Seguridad y } \\
\text { disponibilidad } \\
\text { de las } \\
\text { figuras de } \\
\text { apego }\end{array}$} & $\mathrm{DC}$ & 101 & 4,499 & 4.630 & .432 & 40.958 & $.000^{* * *}$ & $\mathrm{PER} \neq \mathrm{DS}$ & -.44 \\
\hline & DS & 162 & 4,499 & 4.686 & .436 & 40.958 & $.000^{* * *}$ & $\mathrm{DBR} \neq \mathrm{DC}$ & -.70 \\
\hline & DBR & 114 & 4,499 & 4.273 & .578 & 40.958 & $.000^{* * *}$ & $\mathrm{DBR} \neq \mathrm{DS}$ & -.83 \\
\hline & MOD & 45 & 4,499 & 3.641 & .793 & 40.958 & $.000^{* * *}$ & $\mathrm{MOD} \neq \mathrm{PER}$ & -1.32 \\
\hline & PER & 78 & 4,499 & 4.478 & .535 & 40.958 & $.000^{* * *}$ & $\mathrm{MOD} \neq \mathrm{DBR}$ & -.99 \\
\hline & & & & & & & & $\mathrm{MOD} \neq \mathrm{DC}$ & -1.76 \\
\hline & & & & & & & & $\mathrm{MOD} \neq \mathrm{DS}$ & -1.97 \\
\hline \multirow{6}{*}{$\begin{array}{l}\text { Preocupación } \\
\text { familiar }\end{array}$} & $\mathrm{DC}$ & 101 & 4,493 & 3.797 & .661 & 11.438 & $.000^{* * *}$ & $\mathrm{DC} \neq \mathrm{MOD}$ & .86 \\
\hline & DS & 158 & 4,493 & 3.644 & .774 & 11.438 & $.000^{* * *}$ & $\mathrm{DC} \neq \mathrm{PER}$ & .74 \\
\hline & DBR & 113 & 4,493 & 3.303 & .704 & 11.438 & $.000^{* * *}$ & $\mathrm{DC} \neq \mathrm{DBR}$ & .73 \\
\hline & MOD & 44 & 4,493 & 3.159 & .907 & 11.438 & $.000^{* * *}$ & $\mathrm{DS} \neq \mathrm{MOD}$ & .60 \\
\hline & PER & 78 & 4,493 & 3.288 & .721 & 11.438 & $.000^{* * *}$ & $\mathrm{DS} \neq \mathrm{PER}$ & .47 \\
\hline & & & & & & & & $\mathrm{DS} \neq \mathrm{DBR}$ & .46 \\
\hline \multirow{5}{*}{$\begin{array}{l}\text { Traumatismo } \\
\text { infantil }\end{array}$} & $\mathrm{DC}$ & 105 & 4,504 & 2.017 & .824 & 10.083 & $.000^{* * *}$ & $\mathrm{PER} \neq \mathrm{DC}$ & -.46 \\
\hline & DS & 158 & 4,504 & 1.579 & .671 & 10.083 & $.000^{* * *}$ & $\mathrm{PER} \neq \mathrm{MOD}$ & -.72 \\
\hline & DBR & 118 & 4,504 & 1.893 & .647 & 10.083 & $.000^{* * *}$ & $\mathrm{DS} \neq \mathrm{DC}$ & -.60 \\
\hline & MOD & 45 & 4,504 & 2.164 & .752 & 10.083 & $.000^{* * *}$ & $\mathrm{DS} \neq \mathrm{MOD}$ & -.85 \\
\hline & PER & 79 & 4,504 & 1.675 & .643 & 10.083 & $.000^{* * *}$ & $\mathrm{DS} \neq \mathrm{DBR}$ & -.48 \\
\hline \multirow[t]{5}{*}{ Resiliencia } & $\mathrm{DC}$ & 98 & 4,488 & 3.732 & .646 & 4.983 & $.001^{* *}$ & $\mathrm{DS} \neq \mathrm{MOD}$ & .50 \\
\hline & DS & 155 & 4,488 & 3.881 & .595 & 4.983 & $.001^{* *}$ & $\mathrm{DS} \neq \mathrm{DBR}$ & .54 \\
\hline & DBR & 112 & 4,488 & 3.567 & .575 & 4.983 & $.001^{* *}$ & & \\
\hline & MOD & 44 & 4,488 & 3.586 & .592 & 4.983 & $.001^{* *}$ & & \\
\hline & PER & 80 & 4,488 & 3.727 & .649 & 4.983 & $.001^{* *}$ & & \\
\hline
\end{tabular}

Nota. $* *=p<.01 ; * * *=p<.001$ 
Tabla 4. Diferencias en la implicación en acoso escolar, en el tipo de apego y en la capacidad resiliente en función del estilo educativo paterno.

\begin{tabular}{|c|c|c|c|c|c|c|c|c|c|}
\hline & Grupos & $N$ & G.L & Media & D. T & $F$ & Sig. & Post Hoc & $D$ de Cohen \\
\hline \multirow[t]{5}{*}{ Agresión } & $\mathrm{DC}$ & 97 & 4,475 & .323 & .406 & 6.704 & $.000^{* * *}$ & $\mathrm{DS} \neq \mathrm{DBR}$ & -.43 \\
\hline & DS & 106 & 4,475 & .228 & .339 & 6.704 & $.000^{* * *}$ & $\mathrm{DS} \neq \mathrm{MOD}$ & -.28 \\
\hline & DBR & 160 & 4,475 & .468 & .465 & 6.704 & $.000^{* * *}$ & & \\
\hline & MOD & 95 & 4,475 & .397 & .449 & 6.704 & $.000^{* * * *}$ & & \\
\hline & I & 18 & 4,475 & .621 & .682 & 6.704 & $.000^{* * *}$ & & \\
\hline \multirow[t]{5}{*}{ Victimización } & $\mathrm{DC}$ & 97 & 4,475 & .634 & .735 & 3.430 & $.009^{* *}$ & $\mathrm{DS} \neq \mathrm{DBR}$ & .34 \\
\hline & DS & 106 & 4,475 & ,438 & .470 & 3.430 & $.009^{* *}$ & & \\
\hline & DBR & 160 & 4,475 & .621 & .592 & 3.430 & $.009^{* *}$ & & \\
\hline & MOD & 95 & 4,475 & .562 & .510 & 3.430 & $.009^{* *}$ & & \\
\hline & I & 18 & 4,475 & .913 & .707 & 3.430 & $.009^{* *}$ & & \\
\hline Seguridad y & $\mathrm{DC}$ & 92 & 4,456 & 4.714 & .416 & 43.820 & $.000^{* * *}$ & $\mathrm{MOD} \neq \mathrm{I}$ & .78 \\
\hline disponibilidad & DS & 103 & 4,456 & 4.755 & .355 & 43.820 & $.000^{* * * *}$ & $\mathrm{DBR} \neq \mathrm{MOD}$ & .79 \\
\hline de las & DBR & 155 & 4,456 & 4.490 & .483 & 43.820 & $.000^{* * * *}$ & $\mathrm{DBR} \neq \mathrm{I}$ & 1.84 \\
\hline figuras de & MOD & 91 & 4,456 & 4.058 & .642 & 43.820 & $.000^{* * *}$ & $\mathrm{DS} \neq \mathrm{MOD}$ & -1.37 \\
\hline \multirow[t]{5}{*}{ apego } & I & 16 & 4,456 & 3.544 & .786 & 43.820 & $.000^{* * *}$ & $\mathrm{DS} \neq \mathrm{DBR}$ & .61 \\
\hline & & & & & & & & $\mathrm{DS} \neq \mathrm{I}$ & -2.81 \\
\hline & & & & & & & & $\mathrm{DC} \neq \mathrm{DBR}$ & .49 \\
\hline & & & & & & & & $\mathrm{DC} \neq \mathrm{MOD}$ & 1.22 \\
\hline & & & & & & & & $\mathrm{DC} \neq \mathrm{I}$ & 2.43 \\
\hline Preocupación & $\mathrm{DC}$ & 90 & 4,449 & 3.825 & .732 & 6.799 & $.000^{* * * *}$ & $\mathrm{DC} \neq \mathrm{MOD}$ & .79 \\
\hline \multirow[t]{4}{*}{ familiar } & DS & 103 & 4,449 & 3.483 & .799 & 6.799 & $.000^{* * *}$ & $\mathrm{DC} \neq \mathrm{DS}$ & .45 \\
\hline & DBR & 154 & 4,449 & 3.441 & .722 & 6.799 & $.000^{* * * *}$ & $\mathrm{DC} \neq \mathrm{DBR}$ & .53 \\
\hline & MOD & 85 & 4,449 & 3.256 & .724 & 6.799 & $.000^{* * *}$ & & \\
\hline & I & 18 & 4,449 & 3.342 & 1.007 & 6.799 & $.000^{* * *}$ & & \\
\hline Traumatismo & $\mathrm{DC}$ & 95 & 4,461 & 1.901 & .824 & 11.985 & $.000^{* * * *}$ & $\mathrm{DBR} \neq \mathrm{MOD}$ & -.54 \\
\hline \multirow[t]{4}{*}{ infantil } & DS & 104 & 4,461 & 1.451 & .507 & 11.985 & $.000^{* * * *}$ & $\mathrm{DS} \neq \mathrm{DC}$ & -.67 \\
\hline & DBR & 155 & 4,461 & 1.669 & .583 & 11.985 & $.000^{* * * *}$ & $\mathrm{DS} \neq \mathrm{I}$ & -1.20 \\
\hline & MOD & 91 & 4,461 & 2.008 & .703 & 11.985 & $.000^{* * *}$ & $\mathrm{DS} \neq \mathrm{DBR}$ & -.40 \\
\hline & I & 17 & 4,461 & 2.117 & .830 & 11.985 & $.000^{* * *}$ & & \\
\hline \multirow[t]{7}{*}{ Resiliencia } & $\mathrm{DC}$ & 87 & 4,445 & 3.781 & .642 & 11.969 & $.001^{* *}$ & $\mathrm{MOD} \neq \mathrm{DC}$ & -.53 \\
\hline & DS & 102 & 4,445 & 4.003 & .606 & 11.969 & $.001^{\text {** }}$ & MOD $\neq$ DS & -.93 \\
\hline & DBR & 151 & 4,445 & 3.731 & .589 & 11.969 & $.001^{* *}$ & $\mathrm{MOD} \neq \mathrm{DBR}$ & -.47 \\
\hline & MOD & 88 & 4,445 & 3.464 & .554 & 11.969 & $.001^{* *}$ & $\mathrm{I} \neq \mathrm{DC}$ & -.74 \\
\hline & I & 18 & 4,445 & 3.316 & .610 & 11.969 & $.001^{* *}$ & $\mathrm{I} \neq \mathrm{DS}$ & -1.14 \\
\hline & & & & & & & & $\mathrm{I} \neq \mathrm{DBR}$ & -.71 \\
\hline & & & & & & & & $\mathrm{DBR} \neq \mathrm{DS}$ & .46 \\
\hline
\end{tabular}

Nota. $^{* *}=p<.01 ;^{* * *}=p<.001$

En la dimensión que representa al apego seguro, fueron los adolescentes con padres democráticos supervisores y controladores, los que mayor puntuación obtuvieron, comparados con los de padres democráticos de baja revelación, moderados e indiferentes. Por su parte, el grupo de jóvenes con padres democráticos de baja revelación, mostró una mayor puntuación en apego seguro comparado con los que sus padres fueron clasificados como moderados e indiferentes, así como el grupo de adolescentes con padres moderados comparado con el de padres indiferentes.

Los jóvenes cuyos padres fueron categorizados como democráticos controladores, mostraron las mayores puntuaciones en preocupación familiar, comparados con los de padres moderados, democráticos de baja revelación y democráticos supervisores.

En cuanto al traumatismo infantil, la menor puntuación la obtuvieron el grupo de adolescentes cuyos padres fueron clasificados como democráticos de baja revelación, comparado con el que sus padres fueron categorizados como moderados, y el grupo de jóvenes con padres democráticos su- pervisores, comparado con el de los hijos e hijas de padres de los otros subtipos democráticos e indiferentes.

Finalmente, los grupos de jóvenes con padres moderados e indiferentes, mostraron una significativa menor capacidad resiliente, comparados con todos los grupos de adolescentes cuyos padres ejercían alguna modalidad de estilo democrático. Por su parte, los chicos y chicas con padres democráticos supervisores, obtuvieron una puntuación mayor en resiliencia, comparados con los de padres democráticos de baja revelación.

Coherencia entre el estilo educativo materno y paterno y ajuste psicosocial adolescente

Para evaluar la coherencia entre el estilo educativo de la madre y del padre, se creó una variable compuesta por cinco niveles. El primer nivel categorizaba a los progenitores que tenían el mismo estilo educativo democrático que su pareja, (por ejemplo, la madre democrático controlador y el padre también). Este grupo fue denominado "igual democrático" 
(ID). El siguiente nivel, denominado "democráticos diferentes" (DD), definía a las parejas en las que el subtipo de estilo democrático de la madre, era diferente al del padre (por ejemplo, la madre "democrática de baja revelación" y el padre "democrático supervisor"). El tercer nivel, que se denominó "uno democrático" (UD), englobaba a las parejas en las que uno de los miembros era democrático, y el otro, tenía un estilo diferente al democrático. El nivel posterior describía a los padres y madres que tenían el mismo estilo, pero ninguno de ellos era democrático, es decir, los dos miembros de la pareja eran moderados, ya que los otros estilos no democráticos eran el negligente y el permisivo, y estos no eran comparables entre sí. Este grupo fue denominado "ambos moderados" (AM). El último nivel lo conformaban las parejas que tenían un estilo educativo diferente, sin ser ninguno de ellos democrático. Este grupo se denominó "diferente ninguno democrático" (DND).

Finalmente, los resultados del ANOVA (Ver Tabla 5) también mostraron diferencias significativas entre los grupos establecidos en función de la coherencia entre el estilo educativo de la madre y el padre, en las variables que representaban al apego seguro, preocupado y desorganizado, así como en la resiliencia, pero no en la agresión $\left(\mathrm{F}_{4,472}=.799 ; p=\right.$ $.526)$ y victimización $\left(\mathrm{F}_{4,472}=.036 ; p=.997\right)$ entre iguales.

Las pruebas post-hoc mostraron que en la dimensión que representaba al apego seguro, el grupo igual democrático y democráticos diferentes obtuvieron una significativa mayor puntuación, comparados con el grupo ambos moderados, uno democrático y diferentes ninguno democrático; así como el grupo uno democrático, comparado con los grupos diferente ninguno democrático y ambos moderados.

El grupo ambos moderados mostró una significativa mayor puntuación en preocupación familiar y traumatismo infantil, comparado con el grupo igual democrático y democrático diferente.

Finalmente, el grupo igual democrático reflejó una mayor capacidad resiliente, comparado con el grupo diferente ninguno democrático.

Tabla 5. Diferencias en el tipo de apego y en la capacidad resiliente en función de la coherencia entre el estilo educativo paterno y materno.

\begin{tabular}{|c|c|c|c|c|c|c|c|c|c|}
\hline & Grupos & $N$ & G.L & Media & D. $T$ & $F$ & Sig. & Post Hoc & $D$ de Cohen \\
\hline Seguridad y & ID & 224 & 4,456 & 4.638 & .473 & 31.849 & $.000^{* * * *}$ & $\mathrm{ID} \neq \mathrm{AM}$ & 1.88 \\
\hline disponibilidad & AM & 27 & 4,456 & 3.687 & .744 & 31.849 & $.000^{* * * *}$ & $\mathrm{ID} \neq \mathrm{UD}$ & .51 \\
\hline de las & DD & 68 & 4,456 & 4.647 & .329 & 31.849 & $.000^{* * *}$ & $\mathrm{ID} \neq \mathrm{DND}$ & 1.35 \\
\hline figuras de & UD & 107 & 4,456 & 4.387 & .534 & 31.849 & $.000^{* * * *}$ & $\mathrm{DD} \neq \mathrm{DND}$ & 1.41 \\
\hline \multirow[t]{4}{*}{ Apego } & DND & 29 & 4,456 & 3.945 & .775 & 31.849 & $.000^{* * *}$ & $\mathrm{DD} \neq \mathrm{AM}$ & 2.01 \\
\hline & & & & & & & & $\mathrm{DD} \neq \mathrm{UD}$ & .56 \\
\hline & & & & & & & & $\mathrm{UD} \neq \mathrm{DND}$ & .75 \\
\hline & & & & & & & & $\mathrm{UD} \neq \mathrm{AM}$ & 1.21 \\
\hline Preocupación & ID & 223 & 4,449 & 3.580 & .749 & 4.386 & $.002^{* *}$ & $\mathrm{AM} \neq \mathrm{ID}$ & -.65 \\
\hline \multirow[t]{4}{*}{ familiar } & AM & 27 & 4,449 & 3.092 & .784 & 4.386 & $.002^{* *}$ & $\mathrm{AM} \neq \mathrm{DD}$ & -.69 \\
\hline & DD & 66 & 4,449 & 3.606 & .741 & 4.386 & $.002^{* *}$ & & \\
\hline & UD & 103 & 4,449 & 3.414 & .823 & 4.386 & $.002^{* *}$ & & \\
\hline & DND & 29 & 4,449 & 3.183 & .701 & 4.386 & $.002^{* *}$ & & \\
\hline Traumatismo & ID & 227 & 4,461 & 1.679 & .691 & 3.444 & $.009^{* *}$ & $\mathrm{AM} \neq \mathrm{ID}$ & .64 \\
\hline \multirow[t]{4}{*}{ infantil } & $\mathrm{AM}$ & 27 & 4,461 & 2.125 & .736 & 3.444 & $.009^{* *}$ & $\mathrm{AM} \neq \mathrm{DD}$ & .70 \\
\hline & DD & 68 & 4,461 & 1.694 & .572 & 3.444 & $.009^{* *}$ & & \\
\hline & UD & 109 & 4,461 & 1.809 & .717 & 3.444 & $.009^{* *}$ & & \\
\hline & DND & 29 & 4,461 & 1.924 & .666 & 3.444 & $.009^{* *}$ & & \\
\hline \multirow[t]{5}{*}{ Resiliencia } & ID & 218 & 4,445 & 3.833 & .615 & 4.938 & $.001^{* *}$ & $\mathrm{ID} \neq \mathrm{DND}$ & .76 \\
\hline & AM & 26 & 4,445 & 3.523 & .553 & 4.938 & $.001^{* *}$ & & \\
\hline & DD & 63 & 4,445 & 3.728 & .616 & 4.938 & $.001^{* *}$ & & \\
\hline & UD & 107 & 4,445 & 3.679 & .642 & 4.938 & $.001^{* *}$ & & \\
\hline & I & 30 & 4,445 & 3.373 & .585 & 4.938 & $.001^{* *}$ & & \\
\hline
\end{tabular}

\section{Discusión}

Este estudio se ha llevado a cabo con el fin de indagar en la relación entre los estilos educativos parentales y la implicación en acoso escolar, la resiliencia y el apego, habiendo elegido estas medidas de ajuste por haber sido escasamente exploradas en relación a los estilos parentales. El primer objetivo fue explorar las dimensiones parentales evaluadas, para establecer una tipología de estilos parentales, basada en un enfoque dimensional, desde el que se han tenido en cuenta un amplio número de variables relativas a las conductas y actitudes parentales.

Las correlaciones preliminares indican la existencia de relaciones significativas, aunque bajas o moderadas (Cohen, 1992), entre la mayoría de las dimensiones de estilos parentales. Destacamos la relación positiva entre afecto y comunicación, promoción de autonomía, revelación y humor, así como la escasa relación observada entre afecto y control conductual y control psicológico, que va en la línea de lo que señalan investigaciones previas (García \& Gracia, 2009, 2010; Oliva et al., 2007). Dichos resultados son coherentes con el 
establecimiento de las categorías de estilos parentales a través del procedimiento de clústering (análisis de conglomerados), ya que, si bien, ciertas dimensiones se hallan relacionadas con la principal dimensión de afecto y comunicación, la mayoría de ellas, y el propio afecto y comunicación, resultan independientes a los elementos coercitivos de los estilos parentales (control conductual y psicológico) (Lim y Lim, 2003; Stevens, 1992; Tabachnick y Fidell, 1983; en García y Gracia, 2010).

Los resultados obtenidos a partir del análisis de conglomerados muestran la existencia de cinco categorías parentales, que han sido confirmadas a través de un análisis discriminante. Cuatro de las cinco categorías generadas, fueron denominadas, tanto para las madres como para los padres: estilo democrático controlador, estilo democrático supervisor, estilo democrático de baja revelación y estilo moderado. La última categoría fue denominada, en el caso de la madre, estilo permisivo, y en el caso del padre, estilo indiferente.

Los padres y madres democráticos controladores, obtuvieron una elevada puntuación en las dimensiones afecto y comunicación, humor, auto-revelación y promoción de la autonomía, y las máximas puntuaciones en las dimensiones control conductual y psicológico. Los padres y madres clasificados como democráticos supervisores, mostraron las mayores puntuaciones en afecto y comunicación, promoción de autonomía, auto-revelación materna y humor, unas elevadas puntuaciones en control conductual y las menores puntuaciones en control psicológico. El grupo democrático de baja revelación, presentó unas elevadas puntuaciones en afecto y comunicación, promoción de la autonomía, humor, y control conductual (aunque menores que las de los otros estilos democráticos), unas puntuaciones moderadas en control psicológico y bajas en auto-revelación. El grupo de padres y madres moderados mostró puntuaciones moderadas en todas las dimensiones. El grupo de madres permisivas, presentó puntuaciones elevadas en afecto, promoción de la autonomía y humor, y moderadas hacia bajas en las dimensiones relativas al control y auto-revelación. Finalmente, los padres indiferentes, reflejaron bajas puntuaciones en todas las dimensiones y moderadas en control psicológico.

Aunque no hemos hallado evidencia científica anterior que reproduzca esta misma tipología, en la clasificación de Baumrind (1991), en la de Maccoby y Martin, o en la elaborada recientemente por Oliva y colaboradores (2008) también aparece el estilo democrático, permisivo e indiferente, descritos de forma muy similar a como lo reflejan las dimensiones parentales evaluadas en este estudio. En cuanto al estilo que en esta investigación hemos denominado "moderado", Torío, Peña e Inda (2008) también hallan un grupo de padres que se caracteriza por presentar puntuaciones intermedias en todas las dimensiones, así como Baumrind (1991) quien describió un estilo "suficiente" que caracteriza a padres moderadamente afectuosos y exigentes.

Es destacable el hecho de que la mayoría de las categorías parentales encontradas, se enmarquen dentro del estilo democrático. Dichas categorías, más o menos similares en las dimensiones de afecto y comunicación, promoción de autonomía, control conductual y humor y diferentes con respecto al control psicológico y auto-revelación, confirman la importancia de estas últimas dimensiones y justifican la necesidad de establecer una nueva tipología, que en consonancia con los resultados de estudios anteriores, permitiera estudiar y diversificar el estilo democrático, tan presente en la sociedad occidental actual. En este caso, la etiqueta añadida a cada estilo democrático permite diferenciarlo de los demás y caracterizarlo. Así, los subtipos controlador y supervisor, aunque se sitúan muy cerca del democrático clásico, reflejan unas elevadas puntuaciones en las dimensiones relativas al control, que no terminan de encajar con el prototipo democrático tradicional definido por Baumrind (1991), más moderado en control. La baja puntuación en auto-revelación del grupo democrático de baja revelación, también lo alejó en parte del estilo democrático puro (Oliva et al., 2008), facilitando así su caracterización y denominación.

Otro elemento a destacar es que el estilo permisivo solo haya aparecido en las madres, y el indiferente en los padres. Dato que es congruente con los resultados de Oliva y colaboradores (2008), quienes también encontraron una mayor frecuencia del estilo indiferente en los padres, y con los de Winsler, Madigan y Aquilino (2005), que demostraron que los padres perciben a sus esposas más permisivas que a ellos mismos. Mestre, Tur, Samper, Nácher y Cortés (2007), también informaron que los adolescentes perciben de sus madres una disciplina más laxa, y por parte de los padres, una mayor ignorancia de sus necesidades. Todo ello parece indicar que la actitud indulgente en la crianza es algo más característica en las madres, mientras que la indiferencia es una actitud más propia de los padres, sin llegar a ser comportamientos exclusivos de dicho género. Probablemente por ello, en nuestra muestra, el insuficiente número de padres y madres enmarcados en estos estilos, ha dificultado la constitución de una agrupación pura, favoreciendo que estos sean asignados a otras categorías más numerosas. Esta misma razón podría estar explicando que no contemos con ninguna tipología cercana al estilo autoritario, que sí está presente en las clasificaciones anteriores, aunque no de forma exacta a las tipologías clásicas, por ello su denominación de "estricto" (Oliva et al., 2008).

Con respecto a nuestro segundo objetivo, los análisis realizados han mostrado la relación entre el estilo educativo parental y las medidas de ajuste psicosocial utilizadas, habiendo aparecido diferencias entre los grupos establecidos sobre el estilo educativo paterno y materno, en la agresión y victimización entre iguales, en la resiliencia, y en las dimensiones que representan al apego seguro, preocupado y desorganizado. Los datos parecen sugerir que el estilo democrático supervisor, tanto en el padre como en la madre, es el que mejor ajuste genera en los hijos, al relacionarse con una menor agresión, victimización y apego desorganizado, y una mayor resiliencia, apego seguro y preocupado, comparado con los otros estilos. El estilo permisivo en la madre, también se ha relacionado con el buen ajuste, al llevar asociada la menor 
media en la dimensión victimización, y una menor puntación en apego preocupado y desorganizado, comparado con el resto de grupos. El peor ajuste se observa, sin embargo, en los hijos e hijas de padres y madres moderados y padres indiferentes, al mostrar una elevada puntuación en agresión, victimización y traumatismo infantil y una baja puntuación en resiliencia y apego seguro. Los adolescentes con progenitores democráticos controladores o democráticos de baja revelación tampoco reflejan un ajuste general totalmente satisfactorio, aunque en algunas dimensiones obtengan indicadores positivos.

Estos resultados confirman nuestra hipótesis e indican, en la línea de la investigación de Parra y Oliva (2006) el efecto perjudicial que un elevado control psicológico y una baja auto-revelación genera en el ajuste adolescente, independientemente de que vayan combinados con un elevado afecto y control conductual, de ahí que el estilo "democrático supervisor" haya sido el más beneficioso, comparado con los otros dos estilos democráticos. Sin embargo, el hecho de que el estilo moderado y el indiferente hayan sido relacionados con el peor ajuste, demuestra, una vez más, la importancia de promover un elevado afecto y comunicación con los hijos e hijas y un moderado control de su conducta. Actitudes parentales, que como los resultados indican, son aún más beneficiosas si van acompañadas de un buen humor, de una elevada promoción de la autonomía y de la conducta de revelación filial, limitando con ello, el uso de estrategias de control manipuladoras.

Dichos resultados son igualmente coherentes con los de Oliva y colaboradores (2008), Baumrind (1991) y Hoeve y colaboradores, (2011) que resaltaban la superioridad del estilo democrático y la inferioridad del estilo indiferente, en la promoción del ajuste psicológico filial; y con los de otros estudios, como el de Pérez (2012), García y Gracia (2009, 2010) y Fuentes et al., (2015) que destacan el efecto beneficioso del estilo permisivo en los adolescentes de nuestro país.

Finalmente, los resultados obtenidos han demostrado la relación existente entre la coherencia en el estilo educativo de ambos progenitores y la resiliencia y el apego seguro, preocupado y desorganizado. Estos datos, que van en la línea de las investigaciones previas (Berkien, et al., 2012; Oliva, et al., 2008; Steinberg, 2001), confirman nuestra hipótesis, indicando que la situación más favorable para el adolescente es que ambos progenitores presenten el estilo democrático, independientemente de si ambos miembros de la pareja tienen el mismo estilo democrático o no. Esta circunstancia se ha relacionado con la mayor capacidad resiliente y apego seguro, un elevado apego preocupado, así como un menor apego desorganizado, comparado con las otras combinaciones de estilos en la madre y el padre. El efecto más perjudicial en el ajuste adolescente se ha advertido cuando ninguno de los padres tenía el estilo democrático, ya sea en la combinación de ambos moderados, o del estilo indiferente y permisivo, combinados entre sí o con el moderado. Dicha situación se ha relacionado con un menor apego seguro, preocupado y resiliencia y mayor apego desorganizado.

Aunque el apego preocupado se ha relacionado de forma significativa con el desajuste adolescente, sobre todo en términos de problemas internalizantes (Cantón y Cortés, 2005), en nuestro estudio, aparece asociado a los estilos parentales o combinaciones de los mismos en la madre y en el padre, que más parecen beneficiar el ajuste psicosocial de los jóvenes. Ello podría estar explicado porque la escala que hemos utilizado para evaluar el apego preocupado, no refleja conductas excesivamente desadaptativas, ya que en general, la mayoría de las cuestiones solo ponen de manifiesto la preocupación por las figuras de apego y la necesidad de estar junto a las mismas. Actitudes, que teniendo en cuenta las moderadas puntuaciones medias que le corresponden, son coherentes con el buen ajuste y con una práctica parental democrática, que fomente la vinculación familiar y el interés por los miembros de la familia.

Este estudio tiene algunas limitaciones relativas a los instrumentos de medida, muestra y diseño que es necesario manifestar. Los adolescentes participantes, no componen una muestra representativa de la población adolescente española, por lo que en una réplica de este trabajo debería ampliarse la muestra y su representatividad. Por otro lado, el uso de autoinformes como instrumento para la recogida de información, al ofrecer la percepción subjetiva de los encuestados, exige ser prudentes en el enunciado de afirmaciones ya que no hablamos de conductas familiares, sino de la percepción filial sobre los estilos educativos parentales. Además, el carácter transversal de esta investigación imposibilita el establecimiento de relaciones causales, aunque permite apuntar la dirección de las mismas.

Como futuras líneas de investigación, proponemos la elaboración de un estudio longitudinal que permita conocer la evolución del estilo educativo parental con respecto al crecimiento y desarrollo de los hijos e hijas y la inclusión de otras medidas de ajuste, centradas en problemáticas actuales que afectan especialmente a los adolescentes, destacando entre ellas el cyberbullying.

Agradecimientos.- Al Ministerio de Educación, Cultura y Deporte del Gobierno de España, por la concesión de la beca FPU que disfruta la primera autora y a los proyectos de investigación que han apoyado la realización de este trabajo: 1) Violencia Escolar y Juvenil: riesgos del cortejo violento, la agresión sexual y el cyberbullying; (VIEJUCO: Ps-2010-17246); 2) Sexting, ciberbullying y riesgos emergentes en la red: claves para su comprensión y respuesta educativa" (EDU2013-44627-P), ambos financiados por el Plan Nacional de Investigación I +D del Gobierno de España. 


\section{Referencias}

Alonso, J., \& Román, J. M. (2005). Prácticas educativas familiares y autoestima. Psicothema, 17(1), 76-82.

Baldry, A. C., \& Farrington, D. P. (2005). Protective factors as moderators of risk factors in adolescence bullying. Social Psychology of Education, 8, 263-284.

Balluerka, N., Lacasa, F., Gorostiaga, A., Muela, A., \& Pierrehumbert, B. (2011). Versión reducida del cuestionario CaMir (CaMir-R) para la evaluación del apego. Psicothema, 23(3), 486-494.

Baumrind, D. (1968). Authoritarian Vs. Authoritative parental control. Adolescence, 3(11), 255-272.

Baumrind, D. (1991). The influence of parenting style on adolescent competence and substance use. Journal of Early Adolescence, 11(1), 56-95.

Berkien, M., Louwerse, A., Verhulst, F., \& Van der Ende, J. (2012). Children's perceptions of dissimilarity in parenting styles are associated with internalizing and externalizing behavior. European Child and Adolescent Psychiatry, 21, 79-85.

Besharata, M. A., Azizia, K., \& Poursharifib, H. (2011). The relationship between parenting styles and children's academic achievement in a sample of Iranian families. Procedia Social and Behavioral Sciences, 15, 12801283

Brighi, A., Ortega, R., Scheitauer, H., Smith, P. K., Tsormpatzoudis, C., Barkoukis, V., Del Rey, R., et al. (2012). European Bullying Intervention Project Questionnaire (EBIPQ). University of Bologna.

Cantón, J., \& Cortés, M. R. (2005). El apego del niño a sus cuidadores. Madrid: Alianza.

Cea d' Ancona, M. A. (2004). Análisis multivariable. Teoría y práctica en la investigación social. Madrid: Síntesis.

Cohen, J. (1992). A power primer. Psychological Bulletin, 112(1), 155-159.

Connor, K. M., \& Davidson, J. R. T. (2003). Development of a new resilience scale: The Connor-Davidson Resilience Scale (CD-RISC). Depression and Anxiety, 18, 76-82.

Deutsch, A. R., Crockett, L. J., Wolff, J. M., \& Russell, S. T. (2012). Parent and Peer Pathways to Adolescent Delinquency: Variations by Ethnicity and Neighborhood Context. Journal of Youth and Adolescence, 41, 10781094.

Frey, A., Ruchkin, V., Martin, A., \& Schwab-Stone, M. (2009). Adolescents in Transition: School and Family Characteristics in the Development of Violent Behaviors Entering High School. Child Psychiatry \& Human Development, 40, 1-13.

Fuentes, M. C., García, F., Gracia, E., \& Alarcón, A. (2015). Los estilos parentales de socialización y el ajuste psicológico. Un estudio con adolescentes españoles. Revista de Psicodidáctica, 20(1).

García, F., \& Gracia, E. (2009). Is always authoritative the optimum parenting style? Evidence from Spanish families. Adolescence, 44(173), 101131.

García, F., \& Gracia, E. (2010). ¿Qué estilo de socialización parental es el idóneo en España? Un estudio con niños y adolescentes de 10 a 14 años. Infancia y Aprendizaje, 33(3), 365-384.

Gómez-Ortiz, O., Del Rey, R., Casas, J. A., \& Ortega-Ruiz, R. (2014). Estilos parentales e implicación en bullying. Cultura \& Educación, 26(1), 132-158.

Hoeve, M., Dubas, J. S., Gerris, J. R. M., Van del Laan, P. H., \& Smeenk, W. (2011). Maternal and paternal parenting styles: Unique and combined links to adolescent and early adult delinquency. Journal of Adolescence, 34, 813-827.

Kawabata, Y., Alink, L. R. A., Tsen, W., Van Ijzendoorn, M. H., \& Crick, N. R. (2011). Maternal and paternal parenting styles associated with relational aggression in children and adolescents: A conceptual analysis and meta-analitic review, Developmental Review, 31, 240-278.

Maccoby, E. E., \& Martin, J. A. (1983). Socialization in the context of the family: Parent-child interaction. In P. H. Mussen \& E. M. Hetherington (Eds.), Handbook of child psychology: Vol. 4. Socialization, personality and social development ( $4^{\mathrm{a}}$ ed. ed., pp. 1-101). New York: Wiley.

Masten, A., \& Powell, J. L. (2003). A resilience framework for research, policy, and practice. In S. S. Luthar (Ed.), Resilience and vulnerability. Adaptation in the context of childhood adversisties (pp. 1-25). Cambridge, R. U.: Cambridge University Press.

Mestre, M. V., Tur, A. M., Samper, P., Nácher, M. J., \& Cortés, M. T. (2007). Estilos de crianza en la adolescencia y su relación con el comportamiento prosocial. Revista Latinoamericana de Psicología, 39(2), 211-225.

Montero, I., \& León, O. G. (2007). A guide for naming research studies in Psychology. International Journal of Clinical and Health Psychology, 7(3), 847862.

Notario-Pacheco, B., Solera-Martínez, M., Serrano-Parra, M. D., BartoloméGutiérrez, R., García-Campayo, J., \& Martínez-Vizcaíno, V. (2011). Reliability and validity of the spanish version of the 10-item ConnorDavidson Resilience Scale (10 items CD-RISC) in young adults. Health and Quality of Life Outcomes, 9(63), 1-6.

Oliva, A., Parra, A., \& Arranz, E. (2008). Estilos relacionales parentales y ajuste adolescente. Infancia y Aprendizaje, 31(1), 93-106.

Oliva, A., Parra, A., Sánchez-Queija, I., \& López, F. (2007). Estilos educativos materno y paterno: Evaluación y relación con el ajuste adolescente. Anales de Psicología, 23(1), 49-56.

Parra, A., \& Oliva, A. (2006). Un análisis longitudinal sobre las dimensiones relevantes del estilo parental durante la adolescencia. Infancia $y$ Aprendizaje, 29(4), 453-470.

Pérez, P. M. (2012). La socialización parental en padres españoles con hijos de 6 a 14 años. Psicothema, 24(3), 371-376.

Silk, J. S., Morris, A. S., Kanaya, T., \& Steinberg, L. (2003). Psychological Control and Autonomy Granting: Opposite Ends of a Continuum or Distinct Constructs? Journal of Research on Adolescence, 13(1), 113-128.

Steinberg, L. (2001). We Know some things: Parent-adolescent relationship in retrospect and prospect. Journal of Research in Adolescence, 11, 1-19.

Torío, S., Peña, J. V., \& Inda, M. (2008). Estilos de educación familiar. Psicothema, 20(1), 62-70.

Torío, S., Peña, J. V., \& Rodríguez, M. C. (2008). Estilos educativos parentales. Revisión bibliográfica y reformulación teórica. Teoría Educativa, 20, 151-178.

Winsler, A., Madigan, A. L., \& Aquilino, S. A. (2005). Correspondence between maternal and paternal parenting styles in early childhood. Early Childhood Research Quarterly, 20, 1-12.

Wolfradt, U., Hempel, S., \& Miles, J. N. V. (2003). Perceived parenting styles, depersonalisation, anxiety and coping behaviour in adolescents. Personality and Individual Differences, 34, 521-532.

(Articulo recibido: 02-08-2013; revisado: 07-11-2014; aceptado: 24-11-2014) 